\title{
Surface Morphology of GaN Films Determined From Quantitative X-ray Reflectivity
}

\author{
D Lederman \\ West Virginia University \\ Zhonghai Yu \\ West Virginia University \\ T H. Myers \\ West Virginia University \\ Michelle Richards-Babb \\ West Virginia University, Michelle.Richards-Babb@mail.wvu.edu
}

Follow this and additional works at: https://researchrepository.wvu.edu/faculty_publications

Part of the Chemistry Commons

\section{Digital Commons Citation}

Lederman, D; Yu, Zhonghai; Myers, T H.; and Richards-Babb, Michelle, "Surface Morphology of GaN Films Determined From Quantitative X-ray Reflectivity" (1997). Faculty \& Staff Scholarship. 863.

https://researchrepository.wvu.edu/faculty_publications/863

This Article is brought to you for free and open access by The Research Repository @ WVU. It has been accepted for inclusion in Faculty \& Staff Scholarship by an authorized administrator of The Research Repository @ WVU. For more information, please contact ian.harmon@mail.wvu.edu. 


\section{Surface morphology of GaN films determined from quantitative $x$-ray reflectivity}

Cite as: Appl. Phys. Lett. 71, 368 (1997); https://doi.org/10.1063/1.119539

Submitted: 27 December 1996. Accepted: 17 May 1997. Published Online: 04 June 1998

D. Lederman, Zhonghai Yu, T. H. Myers, and M. R. Richards-Babb

\section{ARTICLES YOU MAY BE INTERESTED IN}

The influence of inversion domains on surface morphology in GaN grown by molecular beam epitaxy

Applied Physics Letters 71, 3486 (1997); https://doi.org/10.1063/1.120367

A positive temperature coefficient of resistivity effect from a paraelectric $\mathrm{Pt} /\left(\mathrm{Ba}_{0.5}, \mathrm{Sr}_{0.5}\right) \mathrm{TiO}_{3} / \mathrm{IrO}_{2}$ thin-film capacitor

Applied Physics Letters 71, 371 (1997); https://doi.org/10.1063/1.119540

The effect of atomic hydrogen on the growth of gallium nitride by molecular beam epitaxy Applied Physics Letters 69, 2731 (1996); https://doi.org/10.1063/1.117693

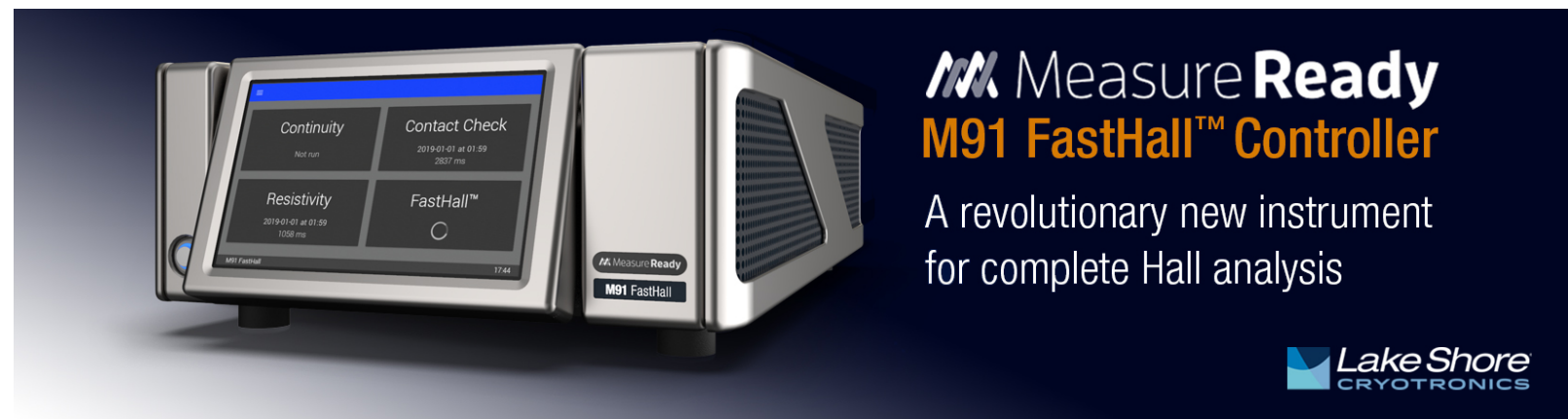




\title{
Surface morphology of GaN films determined from quantitative x-ray reflectivity
}

\author{
D. Lederman, ${ }^{\text {a) }}$ Zhonghai Yu, and T. H. Myers \\ Department of Physics, West Virginia University, Morgantown, West Virginia 26506-6315 \\ M. R. Richards-Babb \\ Department of Chemistry, West Virginia University, Morgantown, West Virginia 26506-6045
}

(Received 27 December 1996; accepted for publication 17 May 1997)

\begin{abstract}
The surface of two GaN films grown under Ga-rich conditions by molecular beam epitaxy was characterized using x-ray reflectivity, assuming a self-affine/fractal surface morphology. The surface height fluctuations were similar for both samples at the largest lateral length scales at which the fractal description is valid, although this lateral length was significantly greater and the "jaggedness" significantly smaller for the sample grown under higher Ga flux. Previous atomic force microscopy images revealed a higher density of large features on the surface for the sample grown under lower Ga flux. The lateral size of the features are dominated by a convolution of the atomic force microscopy tip shape and the actual features on the surface, which precludes an accurate determination of the surface structure at length scales smaller than the tip radius. This study illustrates the importance of using different techniques to evaluate the film surface morphology at different length scales. (C) 1997 American Institute of Physics. [S0003-6951(97)02029-9]
\end{abstract}

It is well known that the surface structure can have a significant impact on a thin film's physical properties. Because of this, a significant portion of the recent work on $\mathrm{GaN}$ thin films has focused on their structural characteristics. Technological applications resulting from GaN films, including optoelectronic devices, will have to overcome constraints resulting from surface defects. Studies have generally focused on the effects of defects on the optical and transport properties of $\mathrm{GaN}$ using a variety of techniques, including $\mathrm{x}$-ray diffraction, atomic force microscopy, scanning electron microscopy, and others.

In this letter, we analyze the X-ray reflectivity (XRR) of GaN films, grown under different Ga flux conditions, using a self-affine/fractal description of the surface. ${ }^{1,2}$ This method has been previously used in for various types of thin films, ${ }^{3-6}$ liquid crystal polymer superlattices, ${ }^{7}$ semiconductor superlattices, $^{8}$ and metallic superlattices. ${ }^{9,10}$ Previously, atomic force microscopy (AFM) studies indicated that Garich molecular beam epitaxy (MBE) growth close to, but below the Ga condensation point, results in smooth, featureless surfaces indicative of two-dimensional growth. ${ }^{11}$ This is consistent with reflection high energy electron diffraction, indicating that Ga-rich conditions are a requirement for twodimensional growth. ${ }^{12}$ In this letter, we study two samples grown under nominally identical conditions except for the Ga flux. Although the surface height fluctuations at the nanometer scale are similar for the two samples $(\approx 2.4 \mathrm{~nm})$, the lateral roughness coherence length is significantly larger and the fractal dimension, or jaggedness, is smaller for the sample grown under a high Ga flux. This agrees with the previous interpretation of the AFM data. AFM images, however, also reveal the presence of large vertical features that tend to mask the surface characteristics at the nanometer length scales. The XRR is insensitive to the large features, but is able to obtain information at small length scales. In

a)Electronic mail: dlederma@wvu.edu general, surface roughness measurements must be performed with different techniques to accurately characterize the surface.

The MBE growth of GaN films has been described previously. ${ }^{11,13}$ Briefly, two samples were grown via MBE on $c$-plane sapphire substrates at $730{ }^{\circ} \mathrm{C}$. A standard MBE source provided the Ga flux. A cryogenically cooled rf plasma source was used to produce the active nitrogen flux. All growth parameters were nominally the same for the films analyzed in this letter, except for their Ga flux. Film GaN1 was grown at a Ga flux of $5 \times 10^{-7}$ Torr (BEP), which represents the onset of Ga-rich growth, while film GaN2 was grown at $7 \times 10^{-7}$ Torr BEP, which is just before the onset of $\mathrm{Ga}$ condensation under these growth conditions.

XRR was measured using a $18 \mathrm{~kW}$ rotating $\mathrm{Cu}$ anode $\mathrm{X}$-ray generator with a four-circle diffractometer in a moderate resolution configuration. ${ }^{14} \mathrm{~A}$ variable slit, placed immediately before the sample, was wide enough to accept the entire width of the specular peak. The angular resolution was $0.12^{\circ}$ for the detector slits and $0.05^{\circ}$ for the sample slits. The slits along the $y$ direction were left wide open, thus averaging over $\mathrm{k}$ space along the $y$ axis. Three types of scans were performed: specular, diffuse near specular, and transverse (rocking curves). For the specular scans, the wave vector transfer $\mathbf{q}$ was perpendicular to the surface normal, for the diffuse near specular scans $\mathbf{q}$ was misaligned by $0.1^{\circ}$ with respect to the specular $\mathbf{q}$, and for the transverse scans the magnitude of $\mathbf{q}$ was constant but its direction rocked about the surface of the sample. The diffuse near specular scan was subtracted from the specular scan to obtain the true specular intensity.

The true specular intensity was fit to a low-angle optical model which takes into account the finite film thickness $\left(t_{f}\right) .^{10,15}$ The analysis was sensitive to both the average surface height fluctuations $\sigma$ and the film thickness. In order to fit the data, the substrate/film interface roughness had to be $\lesssim 0.1 \mathrm{~nm}$, and was therefore fixed at $0.1 \mathrm{~nm}$. The results are 


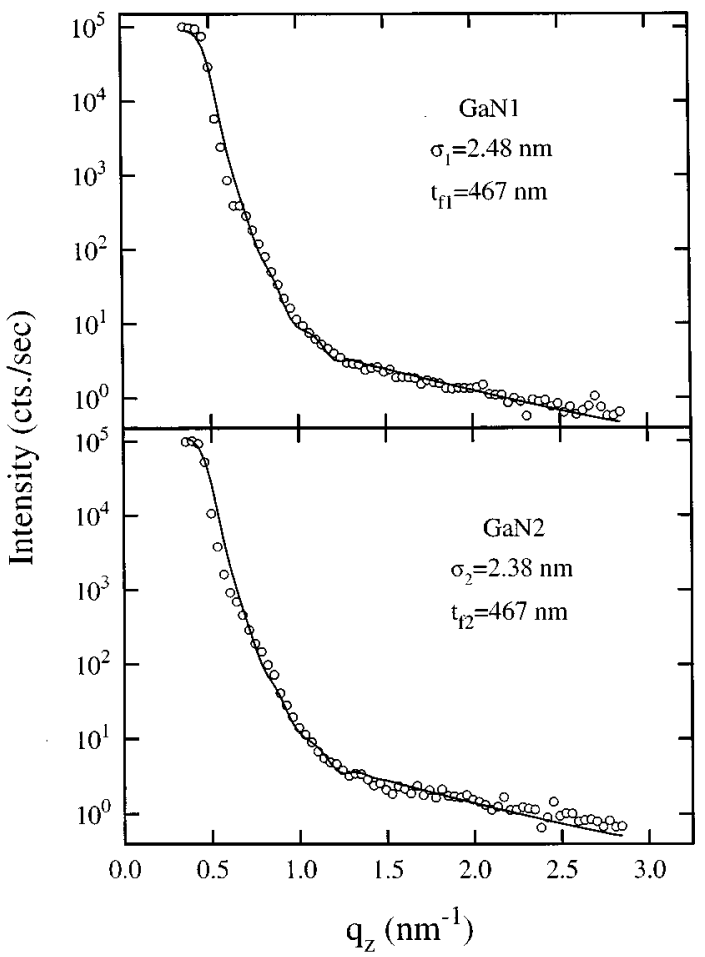

FIG. 1. True specular reflectivity obtained by subtracting a diffuse nearspecular scan from a specular scan for samples GaN1 (low Ga flux) and GaN2 (high Ga flux). The solid curves represent fits to a low angle optical model (Ref. 10). The results for the height fluctuations $\sigma$ and film thickness $t_{f}$ are labeled on the figure.

shown in Fig. 1. The parameters obtained $\left(\sigma_{1}=2.48 \pm 0.1\right.$ $\mathrm{nm}, \quad t_{f 1}=467 \pm 60 \mathrm{~nm} ; \quad$ and $\sigma_{2}=2.38 \pm 0.1 \mathrm{~nm}$, $t_{f 2}=467 \pm 60 \mathrm{~nm}$ for GaN1 and GaN2, respectively) were similar within the numerical uncertainties, determined by monitoring $\chi^{2}$ when the relevant parameter was fixed at different values while the rest were allowed to vary. The fits were significantly improved by taking into account the substrate-epilayer interface, ${ }^{16}$ and hence the results provide a rough estimate of the film thickness. This estimate agrees with the average thickness obtained with contact profilometry at the edge of the film $(700 \mathrm{~nm})$ and transmission electron microscopy micrographs obtained near the center of the film $(230 \mathrm{~nm})$. Finally, the electron density obtained from the fit was within $5 \%$ of the theoretical value calculated from the volume of the unit cell $\left(0.438\right.$ electrons $\left./ \AA^{3}\right)$.

The near-specular and transverse scans were analyzed using the distorted wave Born approximation. ${ }^{2}$ The diffuse scattering function $S_{d}(\mathbf{q})$ (proportional to the intensity) was modeled as

$$
\begin{aligned}
S_{d}(\mathbf{q})= & A \frac{|T(\alpha)|^{2}|T(\beta)|^{2}}{\left|q_{z}^{2}\right|} e^{-0.5 \sigma^{2}\left(\bar{q}_{z}^{2}+\bar{q}_{z}^{* 2}\right)} \\
& \times \int_{-\infty}^{+\infty} d x\left(e^{\left|\bar{q}_{z}\right|^{2} C(x)}-1\right) e^{-i q_{x} x},
\end{aligned}
$$

where $A$ is a scaling factor, $\sigma$ is the average height fluctuation obtained from the true specular data, $T(\alpha)$ is the transmission coefficient of the surface for a grazing angle incidence $\alpha$ ( $\beta$ is the corresponding angle for the outgoing beam), $\bar{q}_{z}$ is the wave vector transfer inside the film. ${ }^{17}$ Equa-

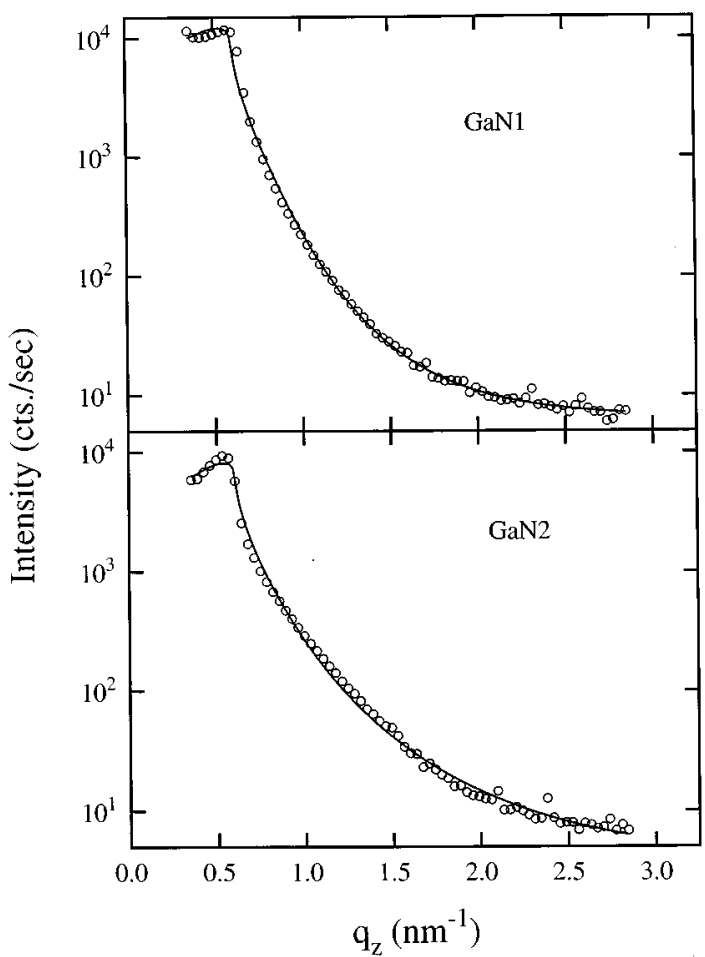

FIG. 2. Diffuse near-specular reflectivity obtained by misorienting the sample by $0.1^{\circ}$ with respect to the specular peak. The solid curves represent fits to Eq. (1). For GaN1, $h=0.40 \pm 0.05, \xi=323.7 \pm 50$, and $\sigma=2.48 \mathrm{~nm}$. For GaN2, $h=0.60 \pm 0.05, \xi=515.7 \pm 50$, and $\sigma=2.38 \mathrm{~nm}$.

tion (1) assumes that the intensity along $q_{y}$ is averaged by keeping the slits in that direction wide open. $C(x)$ is the height-height correlation function, modeled as $C(x)=\sigma^{2} \exp (-|x| / \xi)^{2 h}$, which is applicable to self-affine fractal surfaces with a cutoff. ${ }^{2} \xi$ is the cutoff length of the height fluctuations and $h$ determines the surface "smoothness." Small values of $h$ result in "jagged" surfaces while $h \sim 1$ indicates "smooth" hills and valleys. At small length scales $|x| \leq \xi$, the surface is self-affine/fractal in nature with a fractal dimension $D_{s}=3-h .^{2}$ For $|x| \geqslant \xi$, the fractal height fluctuations saturate at a value equal to $\sigma .^{2,6}$ This could occur because the fractal description is limited by the average film grain size.

Transverse scans were obtained at about $q_{z o}=1.422$ and $q_{z o}=2.134 \mathrm{~nm}^{-1}$ and fit only for $q_{x}>0$ so that the reflected beam was smaller than the detector slit. ${ }^{14}$ The calculation was convoluted with the instrumental resolution, ${ }^{14}$ and the specular peak near $q_{x}=0$ was modeled as a gaussian whose width corresponds to the instrumental resolution. The height of the specular peak was also fit because it includes the sharp contribution from the smooth substrate surface. The best parameters were found by self-consistently fitting Eq. (1), with $\sigma$ fixed to the values obtained from the true specular intensity (Fig. 1), to the transverse and diffuse near-specular scans. The integral in Eq. (1) was calculated numerically by breaking it down into several integrals, ${ }^{18}$ each of which was calculated by decomposing the integrand into Chebyshev polynomials, and each of the Chebyshev terms was calculated by a fast fourier transform. ${ }^{19}$ Hence, there were five fitting parameters: $\xi, h, A$, the height of the specular peak, and a small background intensity. 

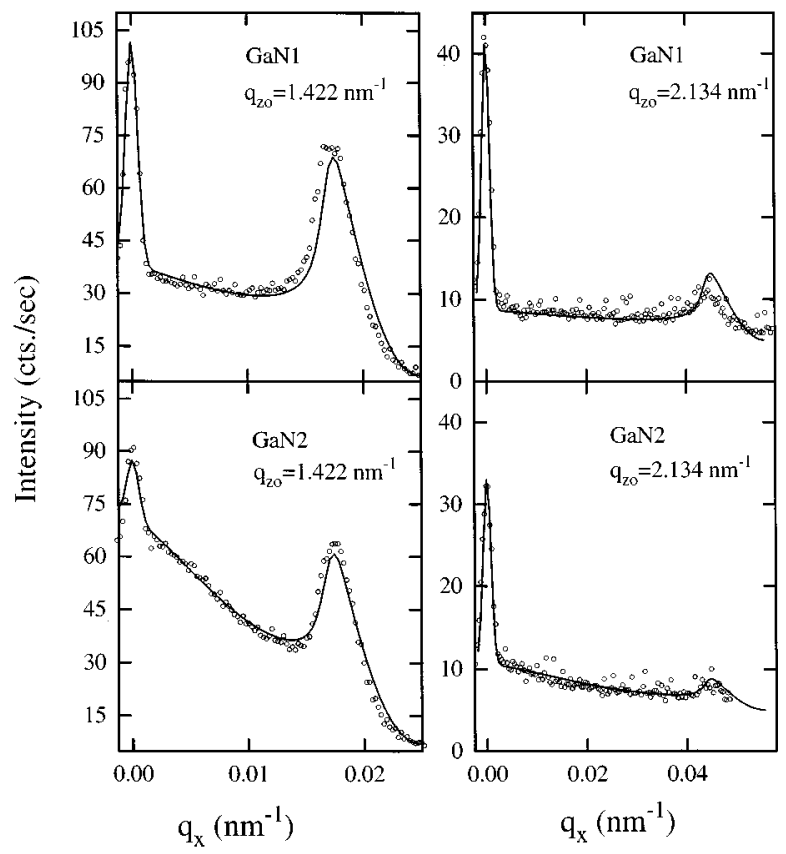

FIG. 3. Transverse (rocking curve) scans measured about $q_{z o}=1.422$ and $q_{z o}=2.134 \mathrm{~nm}^{-1}$. The solid curves represent fits to Eq. (1). For GaN1, $h=0.40 \pm 0.05, \quad \xi=323.7 \pm 50, \quad$ and $\sigma=2.48 \quad \mathrm{~nm}$. For GaN2, $h=0.60 \pm 0.05, \xi=515.7 \pm 50$, and $\sigma=2.38 \mathrm{~nm}$.

The results are shown in Figs. 2 and 3. The peaks at large values of $q_{x}$ in Fig. 3 correspond to angles where $\alpha$ or $\beta$ are close to the critical angle, and are commonly known as "Yoneda wings." ${ }^{20,17}$ For even larger $q_{x}$, the intensity drops because the sample blocks the beam. Qualitatively, the GaN1 transverse scans depend more strongly on $q_{x}$ than the GaN2 scans because the character of their height fluctuations in the lateral direction is different. Quantitatively, the parameters obtained from these fits are $h_{1}=0.40 \pm 0.05$ and $\xi_{1}=323.7 \pm 50 \mathrm{~nm}$; and $h_{2}=0.60 \pm 0.05$ and $\xi_{2}=515.7 \pm 50$ $\mathrm{nm}$. Assuming that $\xi$ is proportional to the average grain size, and because higher values of $h$ mean a lower fractal dimension, sample GaN2, grown at a higher Ga flux, is smoother in the sense that the average grain size is larger and the surface of each individual grain is smoother. The growth of $\mathrm{GaN} 2$ is therefore more two-dimensional than that of GaN1.

Previous qualitative AFM work on these samples ${ }^{11}$ measured large features on the surface, approximately $200 \mathrm{~nm}$ wide and $250 \mathrm{~nm}$ high. The number of features/area was significantly lower for the higher Ga flux sample GaN2. A quantitative analysis of the average height fluctuations between the features found $\sigma=1.6 \pm 0.2 \mathrm{~nm}$ for $\mathrm{GaN} 1$ and $\sigma=1.9 \pm 0.5 \mathrm{~nm}$ for GaN2. The two values of $\sigma$ are the same within their uncertainties, although lower than the value obtained from the XRR $(2.4 \mathrm{~nm})$. This discrepancy could be due to the significant size of the AFM tip used $(200 \mathrm{~nm}$ radius), which combined with the roughness present in the sample, makes a quantitative analysis of the AFM images unreliable without taking into account the shape of the tip. ${ }^{21}$ The x-rays seem to probe the surface morphology between the large features, since the height fluctuations of the two samples was similar, even though the AFM indicated that approximately $5 \%$ of the surface of $\mathrm{GaN1}$ contained the larger features with an average separation of $1000 \mathrm{~nm}$. The $\mathrm{x}$-rays may also probe the surface of these large features, although this contribution is probably small and proportional the surface area of the sample they occupy (i.e., 5\%). These results indicate that a complete surface analysis must include a combination of techniques which are sensitive to roughness at different length scales.

In conclusion, the surface roughness of GaN films grown under different Ga flux conditions was quantitatively analyzed using XRR. Although the two samples used in this study had similar surface height fluctuations along the growth direction, the lateral character of the roughness was different. The sample grown at a higher Ga flux had significantly larger grain sizes and a lower fractal dimension than the sample grown at a lower Ga flux, consistent with increasingly two-dimensional growth. This study confirms prior interpretations of AFM images and presents a new way of analyzing the surface roughness of GaN films. A complete analysis of the surface morphology must include different techniques, such as AFM and XRR, which are sensitive to roughness at different length scales.

The authors thank Dr. Linda Romano at Xerox PARC for the TEM imaging. This work was supported by the Alcoa Foundation (DL) and the Office of Naval Research (TM) under Grant No. N00014-94-1-1149.

${ }^{1}$ For a review of fractal description of surfaces, see Dynamics of Fractal Surfaces, edited by F. Family and T. Vicsek (World Scientific, Singapore, 1991).

${ }^{2}$ S. K. Sinha, E. B. Sirota, S. Garoff, and H. B. Stanley, Phys. Rev. B 38, 2297 (1988)

${ }^{3}$ C. H. Lee, K. S. Liang, F. S. Shieu, S. L. Sass, and C. P. Flynn, Mater. Res. Soc. Symp. Proc. 209, 679 (1991).

${ }^{4}$ C. Thompson, G. Palasantzas, Y. P. Feng, S. K. Sinha, and J. Krim, Phys. Rev. B 49, 4902 (1994).

${ }^{5}$ J. Nogués, D. Lederman, and I. K. Schuller, Appl. Phys. Lett. 68, 3186 (1996).

${ }^{6}$ V. Nitz, M. Tolan, J.-P. Schlomka, O. H. Seeck, J. Stettner, W. Press, M. Stelzle, and E. Sackmann, Phys. Rev. B 54, 5038 (1996).

${ }^{7}$ R. E. Geer, R. Shashidhar, A. F. Thibodeaux, and R. S. Duran, Phys. Rev. Lett. 71, 1391 (1993).

${ }^{8}$ S. K. Sinha, M. K. Sanyal, A. Gibaud, S. K. Satija, C. F. Majkrzak, and H. Homma, in Science and Technology of Nanostructured Magnetic Materials, edited by G. C. Hadjipanayis and G. A. Prinz (Plenum, New York, 1991), p. 145; S. K. Sinha, M. K. Sanyal, S. K. Satija, C. F. Majkrzak, D. A. Neumann, H. Homma, S. Szppala, A. Gibaud, and H. Morkoç, Physica B 198, 72 (1994).

${ }^{9}$ D. E. Savage, J. Kleiner, N. Schimke, Y.-H. Phang, T. Jankowski, J. Jacobs, R. Kariotis, and M. G. Lagally, J. Appl. Phys. 69, 1411 (1991).

${ }^{10}$ E. E. Fullerton, J. Pearson, C. H. Sowers, S. D. Bader, X. Z. Wu, and S. K. Sinha, Phys. Rev. B 48, 17432 (1993).

${ }^{11}$ M. Richards-Babb, S. L. Buczkowski, Z. Yu, and T. H. Myers, Mater. Res. Soc. Symp. Proc. 395, 237 (1996).

${ }^{12}$ T. D. Moustakas, Mater. Res. Soc. Symp. Proc. 395, 111 (1996).

${ }^{13}$ Z. Yu, S. L. Buczkowski, N. C. Giles, T. H. Myers, and M. R. RichardsBabb, Appl. Phys. Lett. 69, 2731 (1996).

${ }^{14}$ J. D. Shindler and R. M. Suter, Rev. Sci. Instrum. 63, 5343 (1992).

${ }^{15}$ E. E. Fullerton, I. K. Schuller, H. Vanderstraeten, and Y. Bruynseraede, Phys. Rev. B 45, 9292 (1992).

${ }^{16}$ B. Vidal and P. Vincent, Appl. Opt. 23, 1794 (1984).

${ }^{17}$ S. K. Sinha, J. Phys. (France) III 4, 1543 (1994).

${ }^{18}$ M. Blakemore, G. A. Evans, and J. Hyslop, J. Comput. Phys. 22, 352 (1976).

${ }^{19}$ W. H. Press, B. P. Flannery, S. A. Teukolsky, and W. T. Vetterling, Numerical Recipes in C: The Art of Scientific Computing (Cambridge University Press, Cambridge, 1992), p. 190.

${ }^{20}$ Y. Yoneda, Phys. Rev. 131, 2010 (1963).

${ }^{21}$ S. M. Paik, S. Kim, and I. K. Schuller, Phys. Rev. B 44, 3272 (1991). 